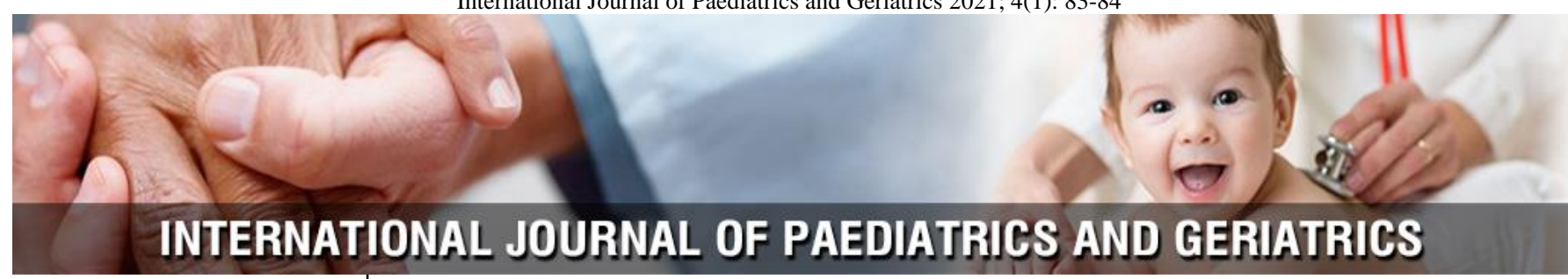

P-ISSN: 2664-3685

E-ISSN: 2664-3693

www.paediatricjournal.com

IJPG 2021; 4(1): 83-84

Received: 15-01-2021

Accepted: 17-02-2021

Dr Amit Vatkar

Consultant, MGM, Medival

College, Navi Mumbai,

Maharashtra, India

Dr Nisha Dolas

Honorary Pediatrician,

Mumbai, Maharashtra, India

Dr Kanmani K

Pediatric Resident, MGM

Medical College, Navi Mumbai,

Maharashtra, India

Dr Vijay Kamale

HOD Department of

Pediatrics, MGM Medical

College, Navi Mumbai,

Maharashtra, India
Corresponding Author:

Dr Amit Vatkar

Consultant, MGM, Medival

College, Navi Mumbai,

Maharashtra, India

\section{Bainbridge-Roper syndrome: A rare case of developmental delay}

\author{
Dr. Amit Vatkar, Dr. Nisha Dolas, Dr. Kanmani K and Dr. Vijay Kamale
}

DOI: $\underline{\text { https://doi.org/10.33545/26643685.2021.v4.i1b.135 }}$

\begin{abstract}
ASXL3 mutations were first identified in 2013 by Bainbridge et al. as a cause of syndromic intellectual disability in four children with similar phenotypes using whole-exome sequencing. The clinical features - postulated by Bainbridge et al. were developmental delay, severe feeding difficulties, failure to thrive and neurological abnormalities. To date, a total of nine individuals with BRPS have been published in the literature in four reports (Bainbridge et al., Dinwiddie et al, Srivastava et al. and Hori et al.). In this report, we describe an uncertain variant present in Exon 11 of ASXL3(+) heterozygous zygosity in a female child with clinical features: truncal muscular hypotonia with significant motor delay, profound speech impairment, intellectual disability and a characteristic dysmorphic facies phenotype (open mouth, full lips, depressed nasal bridge, metopic prominence, microcephaly (45.5.cm), with camptodactyly and dysplastic fingers). Hence, we are reporting a rare case of developmental delay.
\end{abstract}

Keywords: Bainbridge-Roper, neurological abnormalities, developmental delay

\section{Introduction}

Bainbridge-Ropers syndrome (BRPS) is a rare genetic condition, first described in 2013 that results from a change in function of a gene called ASXL3 [3]. LOF (Loss of function) mutations are thought to be responsible for BRPS. Mutations in ASXL3 have also been identified that cause a change in the functional protein but do not cause BRPS or any obvious outcome. About 30 children have been described in the medical literature as having a mutation in one of their two copies of the ASXL3 gene. In 2018 an estimated 200 are known worldwide and many more have yet to be diagnosed ${ }^{[3] .}$

Case Presentation: A 7-year-old female child was brought with complaints of global developmental delay with current milestones of sitting without support transiently, standing with support (gross motor), transfers objects (fine motor), speech is babbling with recognition, regard and social smile present. Child was a Full-term baby born via LSCS in view of preeclampsia. Child cried on stimulation with APGAR score 7 at birth and 8 in 5 mins and was admitted in NICU for a period of 5 days. Child had hypertonia at birth and MRI Brain and EEG at birth were normal. Child then had a history of 2 episodes of focal type seizures $(\mathrm{R}>\mathrm{L})$ when the child was 3.5 years of age and was started on oral Levetiracetam till 5 years of age. On examination, child had Dysmorphic features like open mouth, full lips, depressed nasal bridge, metopic prominence, microcephaly $(45.5 . \mathrm{cm})$, camptodactyly, truncal hypotonia, dysplastic fingers with B/L CTEV and marked ASD features with self-stimulating behaviour. BERA was suggestive of Right ear moderate sensorineural hearing loss and Left ear severe to profound sensorineural hearing loss and was advised on custom made ear mould with hearing aids. MRI Brain was suggestive of a sequalae of Hypoxic ischemic encephalopathy. Chromosomal Microarray analysis was done which showed no pathology. DNA analysis was suggestive of an uncertain variant present in Exon 11 of ASXL3 (+) heterozygous zygosity (BAINBRIDGE ROPERS) along with Exon 2 of ADAT3 (+) homozygous zygosity (Mental retardation-36) and was asked for clinical correlation. Child is currently on speech therapy, occupational therapy and Physiotherapy.

\section{Discussion}

ASXL3, the pathogenic gene of rare BRPS, is a member of the ASXL gene family, located at $18 \mathrm{q} 12.1$, with a length of $6.8 \mathrm{~kb}$, containing 12 exons and encoding the multicomb protein ${ }^{[1]}$. 
The polycarpus family is a group of transcription factors that regulate target genes at the chromatin level through epigenetic modification, and they are involved in embryonic development, cell proliferation and tum or formation. Missense variations in the ASXL3 gene are closely associated with autism spectrum disorders, and dysfunction variants can lead to severe psychomotor retardation. In our case, it is revealed that a de novo heterozygous frameshift variant in exon 11 of the ASXL3 gene is present. The uniqueness is the variant found in this patient is an unidentified and no pathogenic or likely pathogenic variant was found on this particular phenotype. Most mutations are found in one copy of the ASXL3 gene, and most people will also have a second fully functional copy of ASXL3. Typical features of Bainbridge syndrome are seen in our case such as dysmorphic facies, camptodactyly, significant developmental and microcephaly. The prognosis of patients is poor ${ }^{[2]}$. There is no effective treatment for BPRS, so only symptomatic treatment can be conducted. For example, rehabilitation treatment for psychomotor retardation in the early stage is conducted, and the specific effect needs to be verified by long-term follow-up data.

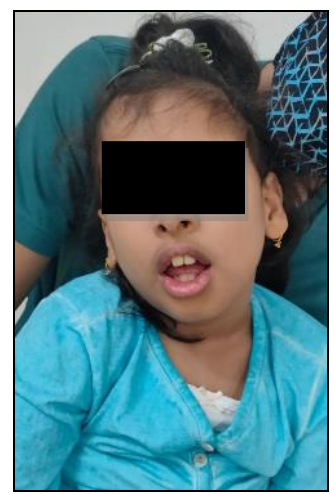

Fig 1: A 7-year-old female child with dysmorphic features



Fig 2: Dysplastic fingers

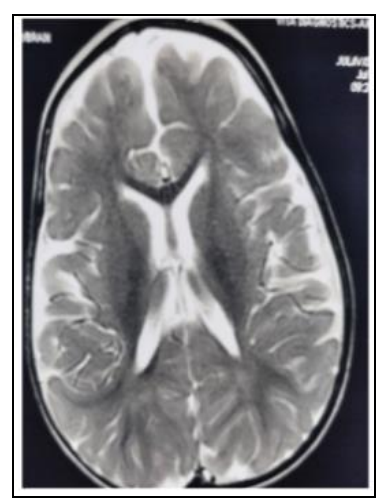

Fig 3: MRI Brain of the child was suggestive of hypoxic ischemic encephalopathy

\section{Conclusion}

The ASXL3 gene is known to instruct different cells in different organs at different stages of development, changes to this gene can therefore affect different parts of the body in different ways. The most important role of ASXL3 is found to be in the brain during early development. Bainbridge-Ropers syndrome is thought to be most often caused by a new (de novo (dn)) mutation in the ASXL3 gene. Mutations in ASXL3 that cause BRPS are not often inherited from a parent, the risk of having another child with this syndrome is therefore thought to be very low. When a child is identified as having an ASXL3 mutation, both parents are usually offered a genetic test to establish possible inheritance. Although it is rare, it is possible for an adult with BRPS, to pass on their genetic variation to their child. Clinical features of BPRS like feeding difficulties, growth disorders, developmental delays and special faces of children, should be identified as soon as possible to improve the genetic examination and clear diagnosis.

\section{References}

1. Linfeng Yang, Bin Guo, Weiwei Zhu, Lei Wang, Bingjuan Han, Yena Che et al. BMC Pediatrics on Bainbridge-ropers syndrome caused by loss-of-function variants in ASXL3: Clinical abnormalities, medical imaging features, and gene variation in infancy of case report 2020.

2. Alma Kuechler, Johanna Christina Czeschik, Elisabeth Graf, Ute Grasshoff, Ulrike Hüffmeier, Tiffany Busa. European Journal of Human Genetics (2017) published online 30 November 2016.

3. Rare chromosomal disorder support group. Registered in England and Wales-Version 1, 2018. 